\title{
Navigation Signal Simulator for Performance Analysis of GNSS Signals
}

\author{
Deepak Mishra \\ Optical and Digital \\ Communication Group (ODCG), \\ Space Applications Centre (SAC)
}

\author{
Sweta Bhardwaj \\ Manipal University, Jaipur, \\ India
}

\author{
Alak Banik \\ Optical and Digital \\ Communication Group (ODCG), \\ Space Applications Centre (SAC)
}

\author{
T.V.S Ram \\ Optical and Digital Communication Group (ODCG), \\ Space Applications Centre (SAC)
}

\author{
Parimal Majithiya \\ Satcom and Navigation Systems Engineering Division \\ (SNSED), Space Applications Centre (SAC)
}

\begin{abstract}
Interoperability and compatibility is the main goal for current GNSS systems. A concept of Global Navigation Satellite System (GNSS) is to use all navigation system together to provide better capabilities compared with those that would be achieved relying solely on one service or signal. Compatibility, on the other hand, assures that existing GNSS signal is not degrading each other below certain threshold. GNSS provider is concerned about their own signal as well as other signals from different service provider for co-existence. For this reason interference analysis of current GNSS signal is the most needed requirement in current scenario.

India is developing its own regional navigation systems named as Indian Regional Navigation Satellite System (IRNSS).An in-house tool is developed with suitable Graphic User Interface (GUI) which provides static analysis of different type of interference parameters and indicates its compatibility with already existing signals. Using the tool, this paper analyzes the degradation in IRNSS signal performance due to various navigation signals in different bands via consideration of parameters such as Power Spectral Density, Root Mean Square (RMS) Bandwidth and Rectangular Bandwidth. A detailed interference analysis of proposed signals is also calculated.
\end{abstract}

In this paper, an attempt is made to analyze \& review few suitable navigation signals for IRNSS in various navigation bands.

\section{Keywords}

PSD, GNSS, SSC, IRNSS, GPS, RMS.

\section{INTRODUCTION}

Global Navigation Satellite System include network of earth orbiting satellites that broadcast signals used in positioning, navigation and timing services, anywhere on the surface of earth. Today two core global navigation satellite systems (GNSS) namely, United States' Global Positioning System (GPS) and the Russian Global Navigation satellite System (GLONASS) are fully operational and the European navigation satellite system GALILEO and Beidou (China) are currently under development. Many regional navigation satellite systems such as IRNSS (India) and QZSS (Japan) are in the process of development. In addition to these, there also exists many satellite based augmentation systems like
GAGAN from India and WAAS from United States of America.

With the increase in number of navigation signals day by day, the performance assessment of existing and planned GNSS signals becomes necessary from compatibility and interoperability [1] point of view.

Interference analysis is being carried out by various GNSS service provider. F. Soualle et al [2] and S. Wallner et al [3] presented the interference analysis between GPS and Galileo signals. Similarly RAN Yi-hang et al [4] presented the details interference analysis between Galileo and Compass navigation systems. There is various other literatures also, where a detailed interference analysis between various GNSS systems is presented [5]

RAN et al [4] discussed the interference analysis of interplex modulation of GALILEO signal in E1 band. The degradation in $\mathrm{C} / \mathrm{No}$ due to other signals is also mention in this paper. The interoperability and compatibility analysis between Compass and Galileo signals is been presented in RAN et al [1]. The paper by Betz [6] presented the detailed fundamentals of different interference parameter. The $\mathrm{PhD}$ thesis by J.A. Avila-Rodriguez [7] presented the detailed analysis of inter and intra systems interference.

IRNSS would provide two services namely, the Standard Positioning Service for civilian users and the Restricted Services for authorized users. IRNSS will transmit signals in L5 (1176.45 MHz) and S band (2492.028 MHz). BPSK (1) would be used for IRNSS Standard Positioning Service (SPS) signal transmission where as Precision Service will use BOC $(5,2)$ modulation. The number of systems that make use of radio frequency spectrum has increased a lot over the last few decades and these developments have crowded the frequency spectrum significantly.

The complete paper is divided in two parts. In first part, we have calculated navigation parameter through software and compare the these parameters of existing GNSS signal from literature $[2,3,4,5,6,7,8,9,10,11,12,15,16,17,18]$ using a comprehensive table of parameters. Considering the L1 and L5 is the future navigation band. In the second part, we have analyzed proposed a few new suitable signal for IRNSS in L1 and L5 band. A detailed interference analysis of the proposed signal is also presented in this paper. 


\section{INTERFERENCE SOFTWARE}

\subsection{Parameters under consideration}

\subsubsection{Power spectral density}

Power spectral density describes the distribution of power of a signal with respect to frequency. A more power of any navigation signal will cause degradation for other navigation signal. The comprehensive Table-1 shows various globally existing signals and power PSD calculated. Fig1, 2 below show the PSD of signals in L5 and S band of IRNSS. Here the signals are placed around a center frequency of $1176.45 \mathrm{MHz}$ and $2492.026 \mathrm{MHz}$ for L5 and S band respectively, which are also the defined centre frequencies for IRNSS in L5 and S band. The total minimum received power of these different navigation signals received at user terminals are in the range between -155 to $-165 \mathrm{dBW}$.

Table 1: Power Spectral Density (PSD) of various GNSS Systems

\begin{tabular}{|l|l|l|l|}
\hline $\begin{array}{l}\text { GNSS } \\
\text { SIGNAL }\end{array}$ & $\begin{array}{l}\text { Signal } \\
\text { Name }\end{array}$ & $\begin{array}{l}\text { Power } \\
\text { (literature)(dBW) }\end{array}$ & $\begin{array}{l}\text { Power } \\
\text { Calculated } \\
\text { using our } \\
\text { Tool } \\
\text { (GUI)(dBW) }\end{array}$ \\
\hline GPS & L1C/A & $-158.5^{[4]}$ & -158.23 \\
\hline GPS & L1C & $-157^{[4]}$ & -157.01 \\
\hline GPS & L1P & $-163^{[4]}$ & -163.21 \\
\hline GPS & L1M & $-157^{[4]}$ & -157.00 \\
\hline GALILEO & E1 OS & $-157^{[4]}$ & -156.98 \\
\hline GALILEO & E1 PRS & $-158^{[4]}$ & -157.95 \\
\hline GLONASS & C/A & $-161^{[7]}$ & -161.00 \\
\hline CODE & & -168.00 \\
\hline GLONASS & P & $-161^{[7]}$ & -163.20 \\
\hline CODE & & $-158^{[22]}$ & -154.02 \\
\hline IRNSS & L5 & $-154^{[22]}$ & \\
\hline & S & & $-163^{[7]}$ \\
\hline
\end{tabular}

\subsubsection{Spectral separation coefficient}

The Spectral Separation Coefficient (SSC) is directly related to the degradation in $\mathrm{C} / \mathrm{No}$ of the desired signal with respect to other GNSS signal. As per ITU Recommendation M.1831 [17] , the total signal to noise degradation experienced by user in the presence of another interfering signal is given by:-

$$
\boldsymbol{\Delta}_{\mathbf{d B}}=\left[1+\frac{I_{O}}{N_{O}+P_{O}}\right]_{d B}{ }^{-----(1)}
$$

Where No is the thermal noise floor ,Po is intra system Interference ,and Io is the external interference. The external interference level is calculated as
$\mathrm{I}_{\mathrm{o}}[\mathrm{dB} / \mathrm{Hz}]=\mathrm{G}_{\mathrm{agg}}[\mathrm{dB}]+\mathrm{P}_{\max }[\mathrm{dBW}]+\mathrm{SSC}[\mathrm{dB} / \mathrm{Hz}]+\mathrm{L}_{\mathrm{x}}[\mathrm{dB}]$

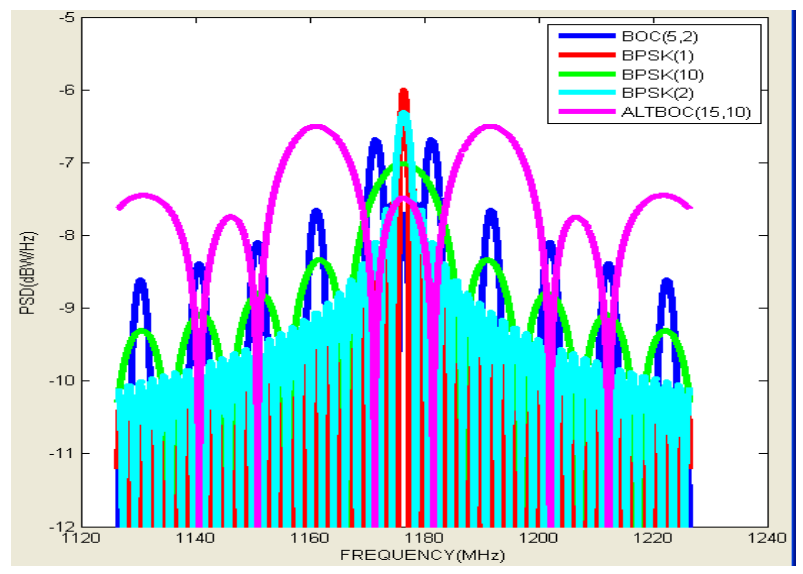

Fig 1: PSD plot of the signals in L5 band

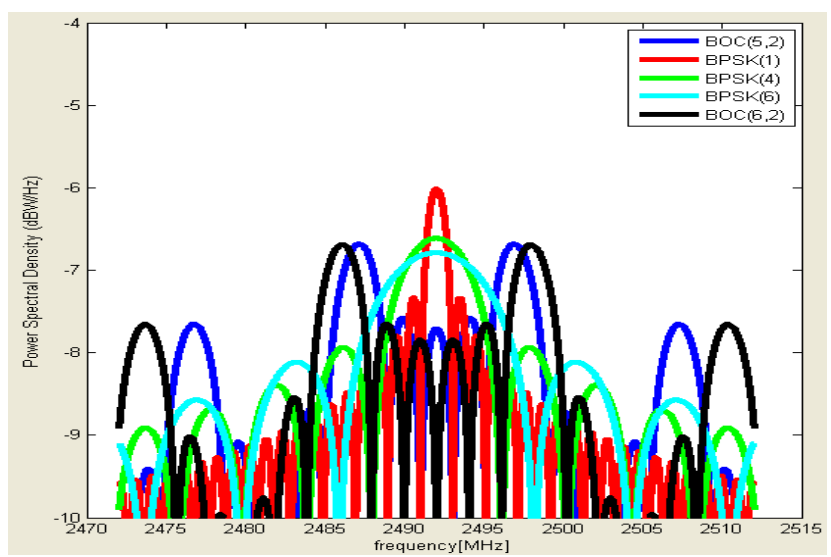

Fig 2: PSD plot of the signals in $\mathrm{S}$ band

$\mathrm{G}_{\mathrm{agg}}$ is the aggregate gain taking into account the interference introduced by all the satellites of one system in view. $\mathrm{P}_{\max }$ is the maximum user receive power, SSC is spectral separation coefficient between interfering and the desired signals. $L_{x}$ is the processing loss.

The SSC is the most widely accepted parameter for interference analysis of GNSS signals. The smaller is the SSC of a modulation with itself the greater is the modulation resistance to multiple access interference with other signals having the same modulation and thus better a modulation can be used for many signals at different received power levels [6].

The expression for SSC can be defined as: -

$$
k_{s s c}=\int_{-\mathfrak{P}_{r} / 2}^{\mathfrak{P}_{r} / 2} \bar{G}_{l}(f) G_{s}(f) d f-
$$

Whereas, $\bar{G}_{l}(\mathrm{f})$ is the normalized power spectral density of composite interference, defined as

$\bar{G}_{l}(f)=\left\{\begin{array}{cr}G_{l}(f) / \int_{-\mathfrak{P}_{t} / 2}^{\mathfrak{P}_{t} / 2} G_{l}(f), & |f| \leq \mathfrak{P}_{t} / 2 \\ 0, & \text { elsewhere }\end{array}\right.$

$\mathfrak{P}_{t}$ is transmitting bandwidth, $G_{s}(f)$ is the normalized PSD, $\mathfrak{P}_{r}$ is the receiver front end bandwidth. Hence, smaller SSC 
also indicates the ability of a modulation to provide enough processing gain against multiple access interference from similar signals so that it can support higher data rates. In general there will be less than $0.1 \mathrm{~dB}$ degradation in $\mathrm{C} / \mathrm{No}$ for SSC below $-60 \mathrm{~dB} / \mathrm{Hz}[8]$.

Table 2, 3 below show the spectral separation coefficient in L5 and S bands respectively, evaluated via in house developed GUI using MATLAB. SSC values shown below, depicts the interference caused to IRNSS signals by its counterpart navigation signals.

\section{Table 2: SSC values in L5 band}

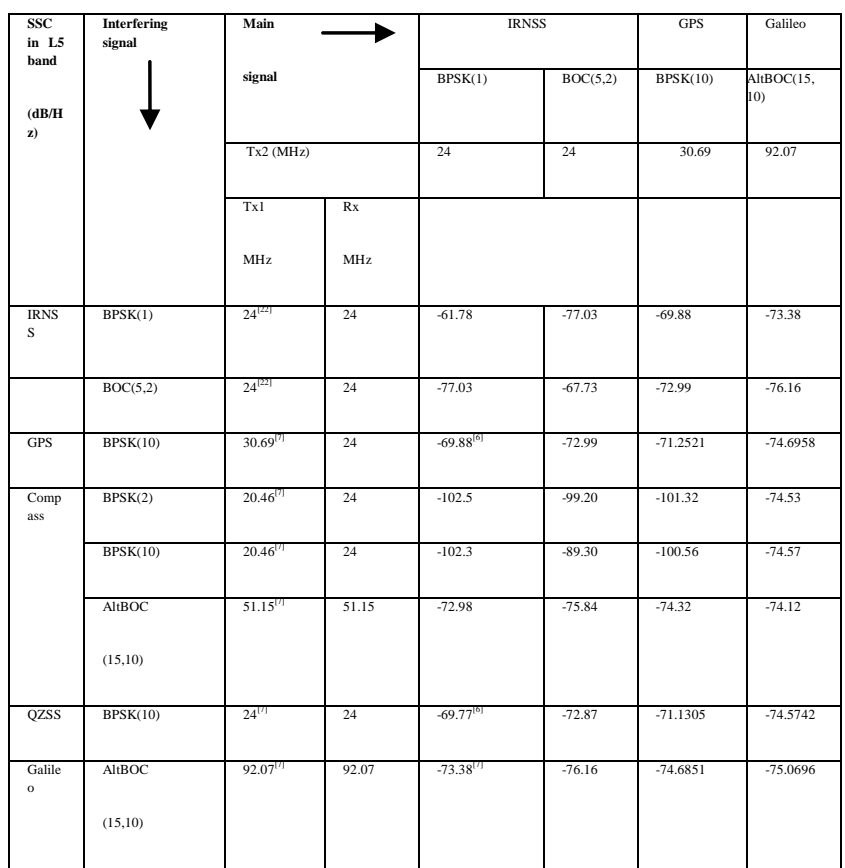

\subsubsection{Root mean square bandwidth:-}

The Root mean square bandwidth is the deciding factor of lower bound on code tracking accuracy [7], as a specific case of the more general lower bound in Gaussian noise and interference having arbitrary spectral shape [10, 11]. RMS bandwidth is also used to decide the front end bandwidth of navigation receiver to select optimum bandwidth in order to minimize the code tracking error performance.

The Root Mean Square Bandwidth (in Hertz) of a band limited signal, denoted by $\overline{\mathfrak{P}}_{r m s}^{2}$ is expressed as [2]: -

$$
\overline{\mathfrak{P}}_{r m s}^{2}=\int_{-\mathfrak{P}_{r} / 2}^{\mathfrak{P}_{r} / 2} f^{2} G_{s}(f) d f \ldots
$$

Whereas $G_{s}(f)$ is the normalized PSD of a given signal, $\mathfrak{P}_{r}$ is the receiver front end bandwidth.
Table 3: SSC values in $\mathrm{S}$ band

\begin{tabular}{|c|c|c|c|c|c|}
\hline \multirow{4}{*}{ 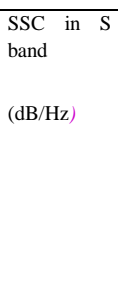 } & \multirow{4}{*}{$\begin{array}{l}\text { Interfering } \\
\text { signal }\end{array}$} & \multirow{2}{*}{\multicolumn{2}{|c|}{ Main signal }} & \multicolumn{2}{|c|}{ IRNSS } \\
\hline & & & & $\operatorname{BPSK}(1)$ & $\operatorname{BOC}(5,2)$ \\
\hline & & \multicolumn{2}{|c|}{ Tx2 (MHz) } & 16.5 & 16.5 \\
\hline & & $\begin{array}{l}\mathrm{Tx} 1 \\
\mathrm{MHz}\end{array}$ & $\begin{array}{l}\mathrm{Rx} \\
\mathrm{MHz}\end{array}$ & & \\
\hline \multirow[t]{2}{*}{ IRNSS } & $\operatorname{BPSK}(1)$ & $16.5^{[22]}$ & 16.5 & -67.77 & -77.01 \\
\hline & $\mathrm{BOC}(5,2)$ & $16.5^{[22]}$ & 16.5 & -77.01 & -61.74 \\
\hline \multirow[t]{3}{*}{ Compass } & BPSK(4) & 16.5 & 16.5 & -66.21 & -77.32 \\
\hline & BPSK $(8)$ & 16.5 & 16.5 & -68.81 & -73.71 \\
\hline & $\operatorname{BOC}(6,2)$ & 16.5 & 16.5 & -82.54 & -70.13 \\
\hline
\end{tabular}

RMS bandwidth is related to Cramer-Rao-Lower bound [5]of any navigation receiver performance and hence it is considered to be an important parameter for performance analysis [8]. This relationship is shown to be

$$
\sigma_{L B}=\frac{1}{2 \pi \overline{\mathfrak{P}}_{r m s}} \sqrt{\frac{B_{L}}{\lambda \frac{C}{N o}}}
$$

Whereas, $B_{L}$ is equivalent rectangular bandwidth, $\frac{C}{N o}$ is a carrier power to noise density ratio.

The frequency-squared term in the RMS bandwidth indicates that even very small amounts of high frequency content in the signal can enable more accurate code tracking. Intuitively, these high frequency components produce sharper edges and more distinct zero crossings in the waveform and gives more accurate code tracking performance. Signals with larger RMS bandwidths offer the potential for more accurate code tracking and better multipath rejection capability.

Fig 3 shows the RMS bandwidth plot of the IRNSS signals in L5 band. It is observed that $\operatorname{BOC}(5,2)$ has higher RMS bandwidth value than BPSK(1) for front-end bandwidth more than $4 \mathrm{MHz}$ It indicates $\operatorname{BOC}(5,2)$ performance is better than $\operatorname{BPSK}(1)$. The BOC $(5,2)$ receiver should be operated for front-end bandwidth of higher than $12 \mathrm{MHz}$, ideal operation range would be 12-28 MHz and 33-28 MHz.

\subsubsection{Rectangular bandwidth:-}

The effective Rectangular Bandwidth of a power spectral density, denoted by $B_{L}$, is given by the bandwidth of a rectangular spectrum having both the same maximum power spectral density and the same area. The expression for Rectangular Bandwidth is shown as [2]:

$\mathfrak{P}_{\text {rect }}=\left[\int_{-\mathfrak{P}_{r} / 2}^{-\mathfrak{P}_{r} / 2} G_{s}(f) d f\right] / k_{(\text {sinusoid at fmax })}$

Whereas $\mathfrak{P}_{r}$ is the front end receiver bandwidth, $k$ is the SSC of a signal with itself. The rectangular has the same significance as that of a noise equivalent bandwidth in communications. Lower the rectangular bandwidth smaller the tracking error.

Table 4 below shows the Rectangular bandwidth of signals in L5 band. 


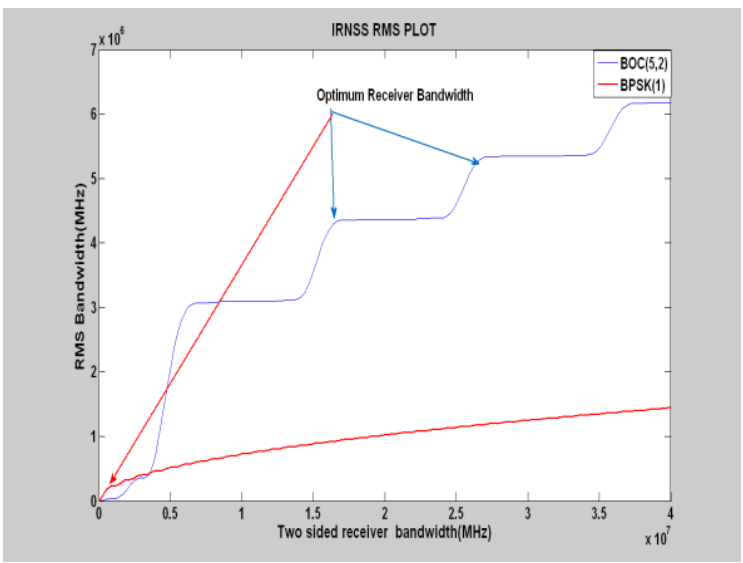

Fig 3: L5 band RMS plot

Table 4: Rectangular bandwidth of the signals in $\mathrm{L} 5$ band

\begin{tabular}{|c|l|c|}
\hline $\begin{array}{c}\text { Navigation } \\
\text { system }\end{array}$ & Signal & $\begin{array}{l}\text { Rectangular } \\
\text { bandwidth(MHz) }\end{array}$ \\
\hline IRNSS & BPSK(1) & 4.919452 \\
\cline { 2 - 3 } & BOC(5,2) & 1.495606 \\
\hline GPS & BPSK(10) & 12.07938 \\
\hline COMPASS & BPSK(2) & 2.886629 \\
\cline { 2 - 3 } & BPSK(10) & 11.32546 \\
\cline { 2 - 3 } & AltBOC(15,10) & 165.7836 \\
\hline GALILEO & AltBOC(15,10) & 216.927 \\
\hline QZSS & BPSK(10) & 11.42157 \\
\hline
\end{tabular}

\section{NEW SIGNAL EXPLORATION}

\subsection{New signal in L1 Band}

MBOC modulation can be considered as an ideal candidate for new IRNSS signal in L1 band. The reason behind selection of MBOC modulation scheme is as follows:-

> Compatibility and interoperability point of view as GPS, GALILEO and COMPASS is already using MBOC modulation.

$>$ Flexibility as MBOC modulation is combination of BOC $(1,1)$ and $\mathrm{BOC}(6,1)$, so in case of large interference more power will be given to $\operatorname{BOC}(6,1)$ modulation scheme for more reliable tracking.

$>$ Flexibility in receiver design as simplest receiver can used only $\mathrm{BOC}(1,1)$ for tracking purpose, while complex receiver use both $\operatorname{BOC}(6,1)$ and $\operatorname{BOC}(1,1)$ for tracking purpose.

$>$ Final but not the least, interference rejection capability of MBOC signal is better as compared to other signals existing in L1 band. Table 5 shows the spectral separation coefficient of $\operatorname{MBOC}(6,1,1 / 11)$ signal at centre frequency $1575.46 \mathrm{MHz}$ with other GNSS signal in L1 band. The rectangular bandwidth of $\operatorname{MBOC}(6,1,1 / 11)$ is mentioned in table 6 .

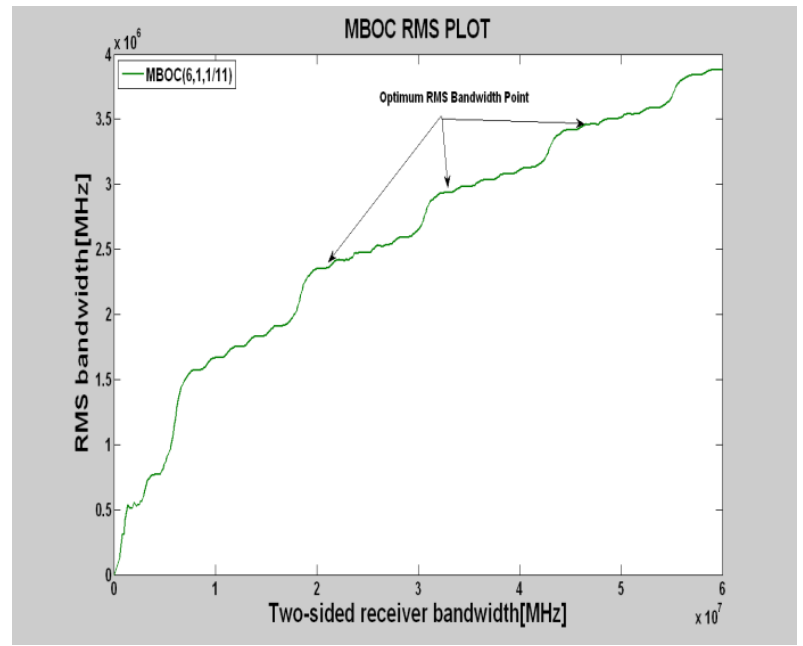

Figure 4: RMS bandwidth of MBOC $(6,1,1 / 11)$

The SSC values indicate that MBOC $(6,1,1 / 11)$ has good interference rejection capability. The RMS plot of MBOC (6, $1,1 / 11$ ) is shown in figure 4.It can be easily seen that around 30 and $40 \mathrm{MHz}$ is the ideal two sided receiver bandwidth for MBOC $(6,1,1 / 11)$ modulation.

\subsection{New signal in L5 Band}

AltBOC $(15,10)$ is considered to be a one of the prominent candidate for future IRNSS signal in L5 band. AltBOC modulation, similar to BOC modulation provides high spectral isolation between the two upper main lobes and the two lower main lobes (considering the I and Q phases separately). This is accomplished by using different codes for each main lobe. The reason behind the selection of AltBOC modulation for

IRNSS in future L5 band is as follows:-

$>$ The Compatibility and interoperability point of view as GALILEO and COMPASS is already using AltBOC $(15,10)$ modulation.

$>$ Two different types of services can be club together in single AltBOC modulation. Hence, it will be an effective utilization of spectrum.

$>$ The interference rejection capability of $\operatorname{AltBOC}(15$, 10) modulation is better as compared to other existing modulation scheme in L5 band.

$>$ Table 7 shows the interference rejection capability in terms of spectral separation coefficient and it can be observed that in all cases its value is always lower than $-60 \mathrm{~dB} / \mathrm{Hz}$. 
Table 5: SSC values in L1 band with Proposed $\operatorname{MBOC}(6,1,1 / 11)$ Modulation

\begin{tabular}{|c|c|c|c|c|}
\hline \multirow{4}{*}{$\begin{array}{l}\mathrm{SSC} \text { in } \\
\mathrm{L1} \text { band } \\
(\mathrm{dB} / \mathrm{Hz})\end{array}$} & \multirow[t]{4}{*}{$\begin{array}{l}\text { Interfering } \\
\text { signal }\end{array}$} & \multirow{2}{*}{\multicolumn{2}{|c|}{$\begin{array}{l}\text { Proposed } \\
\text { signal }\end{array}$}} & $\begin{array}{c}\text { L1 band } \\
(1575.42 \\
\mathrm{MHz})\end{array}$ \\
\hline & & & & $\begin{array}{l}\text { MBOC }(6, \\
1,1 / 1)\end{array}$ \\
\hline & & \multicolumn{2}{|c|}{ Tx2 (MHz) } & 40 \\
\hline & & $\begin{array}{l}\mathrm{Tx} 1 \\
\mathrm{MHz}\end{array}$ & $\begin{array}{l}\mathrm{Rx} \\
\mathrm{MHz}\end{array}$ & \\
\hline IRNSS & MBOC(6,1,1/11) & 40 & 40 & -65.45 \\
\hline \multirow[t]{5}{*}{$\overline{\text { GPS }}$} & BPSK(1) & 30.69 & 40 & -68.15 \\
\hline & BPSK(10) & 30.69 & 40 & -70.44 \\
\hline & TMBOC $(6,1,1 / 11)$ & 30.69 & 40 & -65.40 \\
\hline & $\mathrm{BOC}(1,1)$ & 30.69 & 40 & -65.08 \\
\hline & $\operatorname{BOC}(10,5)$ & 30.69 & 40 & -81.96 \\
\hline \multirow[t]{2}{*}{ Galileo } & $\operatorname{CBOC}(6,1,1 / 11)$ & 40.92 & 40 & -65.45 \\
\hline & $\mathrm{BOC} \cos (15,2.5)$ & 40.92 & 40 & -91.34 \\
\hline \multirow[t]{2}{*}{ Compass } & $\operatorname{BOC}(14,2)$ & 32.74 & 40 & -85.25 \\
\hline & $\operatorname{MBOC}(6,1,1 / 11)$ & 32.74 & 40 & -65.40 \\
\hline
\end{tabular}

*Tx2 is transmitter bandwidth for proposed signal.

*Tx1 is transmitter bandwidth for interfering signal.

* Rx is front end receiver bandwidth.

Table 6: Rectangular Bandwidth in L1 band with Proposed MBOC $(6,1,1 / 11)$ Modulation

\begin{tabular}{|c|c|}
\hline $\begin{array}{c}\text { Proposed signal in L1 } \\
\text { band }\end{array}$ & $\begin{array}{c}\text { Rectangular } \\
\text { Bandwidth(MHz) }\end{array}$ \\
\hline MBOC $(6,1,1 / 11)$ & 3.4346 \\
\hline
\end{tabular}

Figure 5 shows the optimum RMS bandwidth points for AltBOC $(15,10)$. From the figure it is clear that operating point of $\operatorname{AltBOC}(15,10)$ is around two sided receiver bandwidth 51.15 or $92.07 \mathrm{MHz}$. An auto select option is also provided in the GUI which automatically evaluates the optimal transmitter and receiver bandwidth, so there is no need to define transmitter and receiver bandwidth explicitly. For example, In fig 6 BPSK(6) signal is proposed in L5 band at $1191.795 \mathrm{MHz}$ and all the above defined parameters are evaluated using $24 \mathrm{MHz}$ transmitter and receiver bandwidth. An auto mode is provided for evaluation of optimal Tx \& $\mathrm{Rx}$ BW.
Table 7: SSC values of proposed $\operatorname{AltBOC}(15,10)$

Modulation with other existing signals in L5 band

\begin{tabular}{|c|c|c|c|c|}
\hline \multirow[t]{4}{*}{$\begin{array}{l}\text { SSC in } \mathrm{L5} \\
\text { band } \\
(\mathrm{dB} / \mathrm{Hz})\end{array}$} & \multirow[t]{4}{*}{$\begin{array}{l}\text { Interfering } \\
\text { signal }\end{array}$} & \multicolumn{2}{|c|}{$\begin{array}{c}\text { Proposed } \\
\text { signal }\end{array}$} & $\begin{array}{l}\text { L5 Band } \\
\text { (1176.45 } \\
\text { MHz) }\end{array}$ \\
\hline & & & & $\begin{array}{c}\text { AltBOC(1 } \\
5,10)\end{array}$ \\
\hline & & \multicolumn{2}{|c|}{$\mathrm{Tx} 2(\mathrm{MHz})$} & 51.15 \\
\hline & & $\begin{array}{l}\mathrm{Tx1} \\
\mathrm{MHz}\end{array}$ & $\begin{array}{l}\mathrm{Rx} \\
\mathrm{MHz}\end{array}$ & \\
\hline \multirow[t]{3}{*}{ IRNSS } & AltBOC $(15,10)$ & 51.15 & 51.15 & -74.30 \\
\hline & BPSK(1) & 24 & 51.15 & -82.75 \\
\hline & $\mathrm{BOC}(5,2)$ & 24 & 51.15 & -82.46 \\
\hline GPS & BPSK(10) & 30.69 & 51.15 & -82.73 \\
\hline \multirow[t]{3}{*}{ Compass } & BPSK(2) & 20.46 & 51.15 & -95.43 \\
\hline & BPSK(10) & 20.46 & 51.15 & -88.25 \\
\hline & AltBOC $(15,10)$ & 51.15 & 51.15 & -84.46 \\
\hline QZSS & BPSK(10) & 24 & 51.15 & -82.61 \\
\hline Galileo & AltBOC $(15,10)$ & 92.07 & 51.15 & -84.87 \\
\hline
\end{tabular}

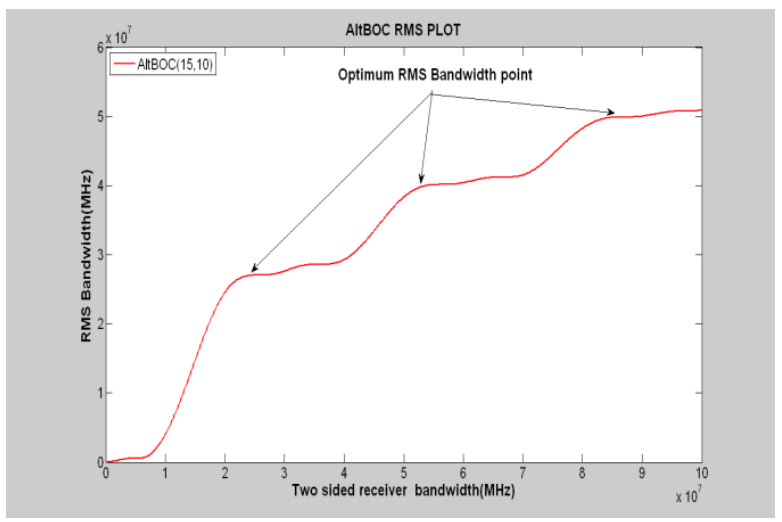

Figure 5: RMS Plot for $\operatorname{AltBOC}(15,10)$

\section{CONCLUSION}

A MATLAB based interference analysis GUI is developed for GNSS signals and the paper has presented a comprehensive list of navigation signal parameters list in terms of 
interference. Using the tool, the static interference has been calculated and studied for presently available various GNSS signal. Further, an attempt is made based on various interference parameters to find out suitable candidate for future IRNSS signals in L1 and L5 band. A provision is made in GUI to analyze any new navigation signal (BOC, BPSK, MBOC, AltBOC modulation) in any navigation band with the user defined transmitter, receiver bandwidth and center frequency. This analysis shows how the performance of a newly proposed signal is affected by already existing signals in the given band. Analyzing the other important parameters defined in section 1 of the paper thoroughly, this tool quickly predict that whether the given modulation is suitable for proposing a signal or not. Considering the importance of interoperability and compatibility for international cooperation and co-existing with GNSS signals from various service providers, further study and research are going to be important question for development and availability of such tool.

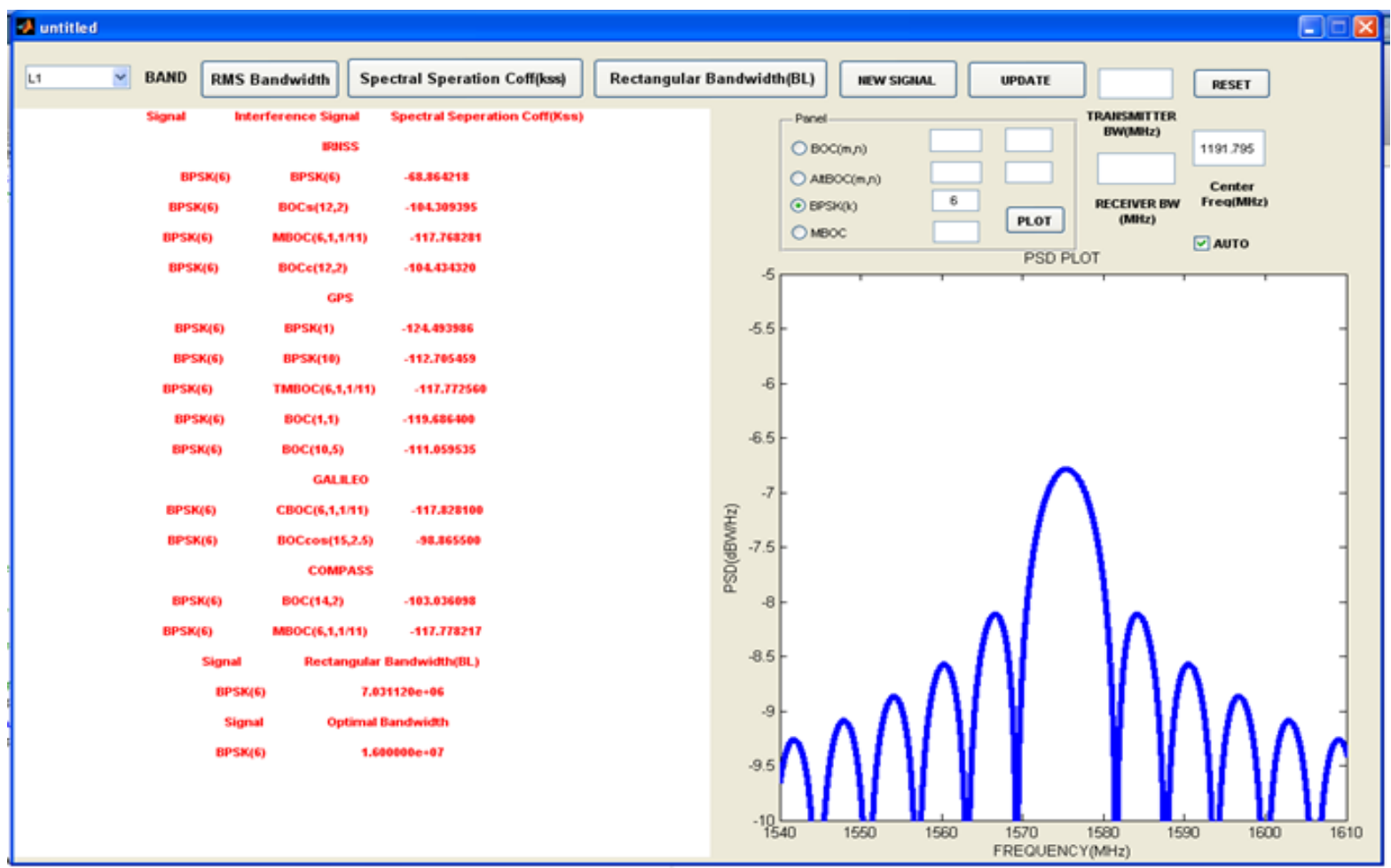

Fig 6 GUI with auto select option analyzing the performance of proposed signal in L5

\section{REFERENCES}

[1] RAN Yi-hang, LIU Yu-qi, HU Xiu-lin, KE Ting, "Evaluation of Intersystem Interference between Compass and Galileo", Journal of Convergence Information Technology, Volume6, Number 6, June 2011

[2] F. Soualle and T. Burger, Interference Analysis for the Global Navigation Satellites Systems Galileo and GPS, AstriumGalileo Phase B2 (post-KPM) document,GAL2ASMD-TN-42154-002, 5.05.2002.

[3] S. Wallner, G.W. Hein, J.-A. Avila Rodriguez, T. Pany, A. Posfay,Interference Computations Between GPS and Galileo, Proceedings of the InternationalTechnical Meeting of the Institute of Navigation, ION-GNSS 2005, 13-16 September, 2005,Long Beach, California, USA.

[4] RAN Yi-hang, LIU Yu-qi, HU Xiu-lin, KE Ting, "Interference Analysis of Interplex Modulation in GALILEO E1 Band", Wireless Communications, Networking and Mobile Computing, 2009. WiCom '09. 5th International Conference , Page(s): 1 - 4 ,E-ISBN :978-1-4244-3693-4,Print ISBN: 978-1-4244-3692-7, INSPEC Accession Number: 10952820 .
[5] Misra, P and Enge, P., "Global Positioning System", Ganga-Jamuna press, New York, 2011

[6] John W Betz, "Binary Offset Carrier Modulations for Radio navigation", Journal of The Institute of Navigation, vol. 48, no.4, winter 2001/2002.

[7] J.A. Avila-Rodriguez, "On generalized signal waveforms for satellite navigation", $\mathrm{PhD}$ dissertation University FAF Munich, Neubiberg, Germany, pp. 408, June 2008.

[8] G. Hein et al, "The New optimized spreading modulation recommended for Galileo L1 OS and GPS L1 C", Proceedings of the IEEE/ION Position, Location and Navigation Symposium, April 2006.

[9] B.W. Parkinson and J.J Spilker, "Global Positioning System: Theory and Applications”, Vol. I, AIAA, 1996.

[10] Wei liu, "Assessing Radio Frequency Compatibility Between Galileo and Compass", Defence Science Journal, 2011.

[11] [GPS ICD 200, 2006] IS-GPS-200 Revision D, IRN200D-001:NAVSTAR GLOBAL POSITIONING 
SYSTEM Navstar GPS Space Segment/Navigation User Interface, dated 7 March 2006.

[12] [GPS ICD-705, 2005] IS-GPS-705, IRN-705-003: Navstar GPS Space Segment/User Segment L5 Interfaces, 22 September 2005.

[13] GPS Interface Specification (ICD), IRN-IS-200F-001: 21st Sept. 2011

[14] GLONASS Interface Control Document, version-5.0, 2002

[15] GALILEO Signal-In-Space, ICD, May 2006

[16] QZSS Interface Specification (ICD), IS-QZSS, ver 1.4, 28th Feb. 2012

[17] International Telecommunication Union Recommendation,ITU-RM,.1831,“Acoordination methodology for RNSS inter-sytem interference estimation," 2007.
[18] J. W. Betz, Major David B. Goldstein, "Candidate Designs for an Additional Civil Signal in GPS Spectral Bands."

[19] Maurizio Fantino, GianlucaMarucco, Paolo Mulassano, Marco Pini, "Performance Analysis of MBOC, AltBOC and BOC Modulations inTerms of Multipath Effects on the Carrier Tracking Loop within GNSS Receivers."

[20] Elliott D. Kaplan, Christopher J. Hegarty, "Understanding GPSPrinciples and Applications, Second Edition".

[21] B. W. Parkinson and J. J. Spilker , "Global Positioning System:Theoryand Applications ,"Vol. 1, AIAA, 1996.

[22] CDR document IRNSS, ISRO-SAC-IRNSS-CDR-01, JUNE 2102 